therefore, to ascertain whether the absorption spectrum described by Cordes and Schenk was to be attributed to the presence of the monoxide.

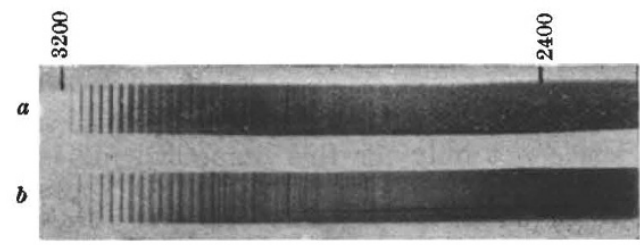

FIG. 1. Absorption spectrum of sulphur dioxide ; $(a)$ before, and $(b)$ after discharge.

An electrodeless discharge was produced in sulphur dioxide and the absorption spectrum was taken before and immediately after the discharge, as shown in Fig. 1. The intensity of the spectra is not quite the same, for a thin sulphur film had partly covered the plate during the $2 \frac{1}{2}$ hours of discharge, and it seemed necessary to take a longer exposure. Nevertheless, in spite of the difference in intensity, the identity of the two spectra cannot be doubted, so that there is definitely no new absorption spectrum produced by the discharge. That is quite clear in the original plate, but naturally not so clear in the reproductions. The presence of sulphur monoxide, on the other hand, in the discharge is proved by its emission spectrum, taken during the discharge (Fig. 2), which shows the same bands as the spectrum that was analysed first by V. Henri ${ }^{4}$ and later by E. V. Martin ${ }^{5}$

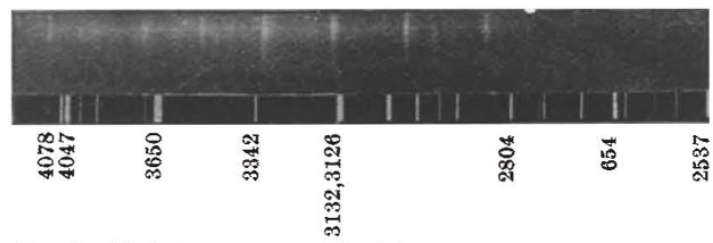

FIG. 2. Emission spectrum of sulphur monoxide, with mercury lines below.

It seems necessary, therefore, to ascribe the absorption spectrum found by Cordes and Schenk to some product of the discharge other than sulphur monoxide ; in other words, our experiment indicates that sulphur monoxide in small concentrations has no absorption spectrum between 3100 and $2500 \mathrm{~A}$.

Physics Department,

G. KornFeld.

University College,

Nottingham. Nov. 23.

${ }^{1}$ G. Kornfeld and M. McCaig, Trans. Farad. Soc., 30, 991 ; 1934 3 . Cordes and $P$. W. Schenk, $Z$. anorg. $u$. allgem. Chem., 214, 33 1933. Z. Elektrochem., 39, 594; 1933. Trans. Farad. Soc., 30, 31 1934.

${ }_{1933}^{3}$. W. Schenk and H. Platz, Z. anorg. u. allgem. Chem., 215, 113

933.

V. Henri and F. Wolff, J. Phys. et Le Radium, VI, 10, 81; 1929

${ }^{5}$ E. V. Martin, Phys. Rev., 41, 167; 1932.

\section{Sources of Error in Absorption Spectroscopy}

In studying the absorption spectra of the vapours of the homologous aldehydes ${ }^{1}$ a band spectrum was observed in butyric and iso-butyric aldehydes, which was ultimately discovered to be due to benzene. Inquiries showed that the catalyst used in the preparation of the aldehydes had been washed with benzene, thus accounting for the presence of an aromatic impurity which would not normally be expected to occur in aliphatic compounds. The spectrum is, however, so intense that, when the observation tube had been used to secure a comparison spectrum of benzene, it was very difficult to get rid of this spectrum in subsequent exposures.

In attempting to photograph the fine structure of acetone, Dr. F. C. Garrow in 1931 observed a very fine series of bands, corresponding in general character with those recorded by Bowen and Thompson ${ }^{2}$, which Norrish, Crone and Saltmarsh ${ }^{3}$ and Noyes, Duncan and Manning ${ }^{4}$ have not been able to reproduce. The bands observed by Dr. Garrow were, however, due to interference, since they were only slightly weakened when the tube was empty; they were also observed with a single end plate, and were only got rid of by using other quartz plates. Bands of a similar character have been described by Schaeffer and others ${ }^{5}$, and those now recorded are probably similar in origin. The occurrence of a few weak bands at wave-lengths less than $2900 \mathrm{~A}$. has already been recorded by us ${ }^{1}$, but no trace of a genuine fine structure could be found in this region.

C. P. SNow.

E. EASTWOOD.

\section{University Chemical Laboratory, Cambridge. Dec. 15 .} 1 Snow and Eastwood, Nature, 133, 908, June 16, 1934.
2 Bowen and Thompson, NATURE, 133, 571, April 14, 1934. 'Bowen and Thompson, Nature, 138, 571, April 14, 1934. ${ }^{3}$ Norrish. Crone and Saltmarsh, J. Chem. Soc., 1458; 1934. ${ }^{5}$ Selényi, Math. u. naturo. Berichte aus Ungarn, 27, 76; 1909. Chinmayanandam, Proc. Roy. Soc, A, 95, 176; 1919. Ghosh, Proc Indian Soc. for Cultivation of Science, 7,$53 ; 1922$. Schaeffer and Fricke, Z. Phy8., 14, 253 ; 1923. Schaeffer, Z. Phys., 17, 155; 1923.

\section{Properties of Liquid Films in Fine-Pored Systems}

ThE properties of liquids, and in particular of water, when held by finely porous material, are of interest to workers in both the pure and applied fields. A growing mass of evidence points to the necessity of assuming a modification in properties of such liquid films even in thicknesses corresponding with many molecular layers.

Shereshefsky ${ }^{1}$ and others have produced definite evidence that measured vapour pressures of liquids in small capillaries (of radius $0.0005 \mathrm{~cm}$. and less) are very much smaller than those calculated from the Kelvin equation. In view of this result, it is interesting to note that experiments at the Building Research Station, shortly to be published, have shown that for several finely pored plastic materials, the hydrostatic suction necessary to remove water is of quite a different order from that which would be expected from the vapour pressure relation. Similar anomalous behaviour has been found when the same moist plastic materials are brought to equilibrium with a solution of sugar of known osmotic pressure through a semi-permeable membrane. Calculations show that large discrepancies also exist in published literature, where it is possible to compare sets of determinations by two dissimilar methods. Thus Szigeti's ${ }^{2}$ measurements of vapour pressures and negative absorptions from sugar solutions show these anomalies.

Among other cases of anomalous behaviour re. ported which may be due to the same causes the following may be noted: Glixelli and Wiertelak ${ }^{3}$ found low values for the electro-kinetic potential in 